\title{
Magnetic fields in our Galaxy on large and small scales
}

\author{
J. L. Han \\ National Astronomical Observatories, Chinese Academy of Sciences \\ Jia-20 DaTun Road, Chaoyang District, Beijing 100012, China \\ email: hjl@bao.ac.cn
}

\begin{abstract}
Magnetic fields have been observed on all scales in our Galaxy, from AU to kpc. With pulsar dispersion measures and rotation measures, we can directly measure the magnetic fields in a very large region of the Galactic disk. The results show that the large-scale magnetic fields are aligned with the spiral arms but reverse their directions many times from the innermost arm (Norma) to the outer arm (Perseus). The Zeeman splitting measurements of masers in HII regions or star-formation regions not only show the structured fields inside clouds, but also have a clear pattern in the global Galactic distribution of all measured clouds which indicates the possible connection of the large-scale and small-scale magnetic fields.
\end{abstract}

Keywords. ISM: magnetic fields, pulsars: general, HII regions, radio lines: ISM, ISM: structure

\section{Introduction}

Inside our Galaxy, the diffuse interstellar medium (ISM), molecular clouds, and very dense cloud core or HII regions, are all permeated by magnetic fields. Many physical processes in the ISM are related to magnetic fields. For example, magnetic fields in the diffuse medium contribute to the hydrostatic balance and stability of the ISM (Boulares \& Cox 1990); magnetic fields are the main agent for transporting charged cosmic-rays(e.g. Tinyakov \& Tkachev 2002, Prouza \& Smída 2003), while magnetic fields in molecular clouds obviously play an important role in the star formation process (Heiles \& Crutcher 2005). However, our knowledge of the Galactic magnetic fields is far from complete.

It is possible that the fields in clouds are enhanced when interstellar gas contracts to form a cloud or the cloud core, so that the observed field strength increases with gas density (Crutcher 1999). Here, I review the observational results of large-scale magnetic fields in our Galaxy mainly revealed by pulsar rotation measures, which are related to the diffuse gas and the spiral structure. Small-scale magnetic fields have been detected by other means, such as continuum polarization surveys of the Galactic plane. I will show that the Galactic distribution of the small scale (AU) magnetic fields detected by maser-lines from clouds are probably related to the large-scale Galactic magnetic field structure. In this paper large scale means a scale larger than the separation of spiral arms, i.e. a scale of $1 \mathrm{kpc}$, while small scale means a smaller scale.

There are five observational tracers for the Galactic magnetic fields: Zeeman splitting, polarized thermal emission from the dusts in clouds, polarization of starlight, synchrotron radio emission, and Faraday rotation of polarized sources. Observations of Zeeman splitting of spectral lines, and of polarized thermal emission from dust at mm, sub-mm or infrared wavelengths, have been used to detect respectively the line-of-sight strength and the transverse orientation of magnetic fields in molecular clouds (e.g. Crutcher 1999, Novak et al. 2003, Fish et al. 2003). Starlight polarization data show that the local field is parallel to the Galactic plane and follows the local spiral arms (see Mathewson \& Ford 
1970, Andreasyan \& Makarov 1989, Heiles 1996a). Since we live near the edge of the Galactic disk, we cannot have a face-on view of the global magnetic field structure in our Galaxy through polarized synchrotron emission, as is possible for nearby spiral galaxies (see reviews by Beck et al. 1996, Beck 2005). Polarization observations of synchrotron continuum radiation from the Galactic disk give the transverse orientation of the field in the emission region and some indication of its strength. Large-angular-scale features are seen emerging from the Galactic disk, for example, the North Polar Spur (e.g. Junkes et al. 1987, Duncan et al. 1997, Duncan et al. 1999, Reich et al. 2002), and the vertical filaments near the Galactic Center (Haynes et al. 1992, Duncan et al. 1998). Faraday rotation of linearly polarized radiation from pulsars and extragalactic radio sources is a powerful probe of the diffuse magnetic field in the Galaxy (e.g. Simard-Normandin \& Kronberg 1980, Han \& Qiao 1994, Han et al. 2006, Brown et al. 2007). Galactic magnetic fields both on large scales and small scales can be revealed by these tracers.

\section{Galactic magnetic fields on large scales}

Our Galaxy is a spiral galaxy. Spiral galaxies have large-scale magnetic fields (Beck 2005). In the last decade, significant progress has been made on revealing the large-scale magnetic fields of our Galaxy in the Galactic central region, in the Galactic halo and the Galactic disk.

Near the Galactic Center, non-thermal gaseous filaments have been discovered (LaRosa et al. 2004, Nord et al. 2004, Yusef-Zadeh et al. 2004). The majority of bright non-thermal filaments are perpendicular to the Galactic plane, indicating predominantly poloidal fields of maybe $\mathrm{mG}$ strength, but some filaments are not, indicating a more complicated field structure than just the poloidal field. LaRosa et al. (2005) detected the diffuse radio emission and argued for a weak pervasive field of tens of $\mu \mathrm{G}$ near the Galactic Center. The new discovery of an infrared 'double helix' nebula (Morris et al. 2006) reinforces the presence of strong poloidal magnetic fields merging from the rotated circumnuclear gas disk near the Galactic Center (Yusef-Zadeh \& Morris 1987). With the development of polarimetry at $\mathrm{mm}$, submm or infrared wavelengths, toroidal fields have been observed in the molecular cloud zone near the Galactic Center (Novak et al. 2003, Chuss et al. 2003), complimenting the poloidal fields shown by the vertical filaments. The large RMs of radio sources near the Galactic Center (Roy et al. 2005) may indicate toroidal field structure.

From the RM distribution in the sky, Han et al. (1997, 1999) identified the striking antisymmetry in the inner Galaxy with respect to the Galactic coordinates after removing the RM "outliers" compared to their neighborhoods. Such an antisymmetry should result from azimuthal magnetic fields in the Galactic halo with reversed field directions below and above the Galactic plane. Such a field can be produced by an 'A0' mode of dynamo. The observed filaments near the Galactic Center should result from the dipole field in this dynamo scenario. The local vertical field component of $0.2 \mu \mathrm{G}$ (Han \& Qiao 1994, Han et al. 1999) may be a part of this dipole field in the solar vicinity. At present, we have observed another 1700 radio sources in the Northern sky by the Effelsberg $100 \mathrm{~m}$ telescope (Han, Reich et al. 2007, in preparation), and wish to do more in the Southern sky with Parkes, so that the rotation measure (RM) sky can be described quantitatively.

Magnetic fields in a large-part of the Galactic disk have been delineated by Faraday rotation data of pulsars, which gives a measure of the line-of-sight component of the magnetic field. Extragalactic sources have the advantage of large numbers but pulsars have the advantage of being spread through the Galaxy at approximately known distances, allowing direct three-dimensional mapping of the magnetic field. Pulsars also give a very 


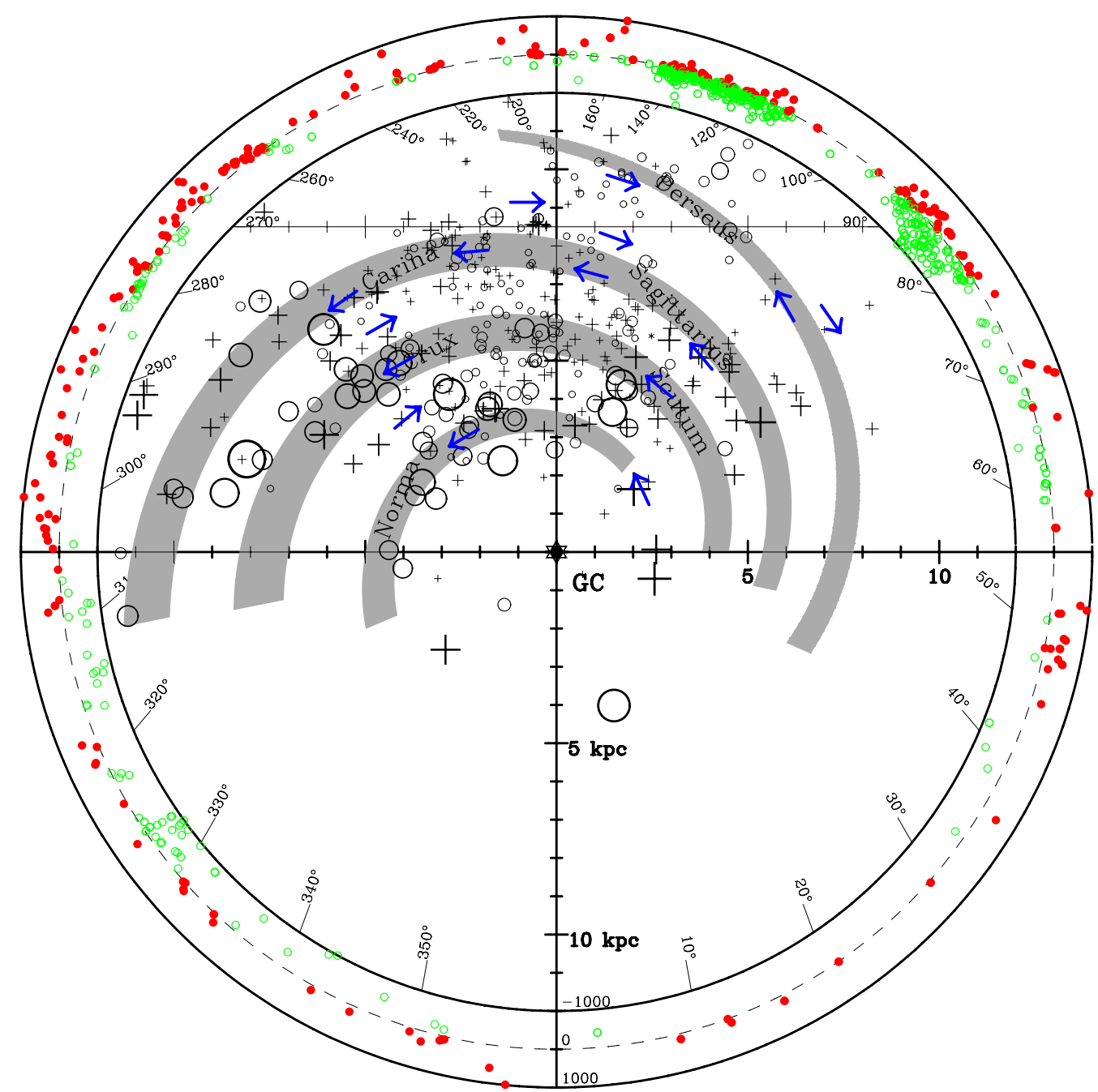

Figure 1. The RM distribution of 374 pulsars with $|b|<8 \mathrm{deg}$, projected onto the Galactic plane. The linear sizes of the symbols are proportional to the square root of the RM values. The crosses represent positive RMs, and the open circles represent negative RMs. The approximate locations of four spiral arms are indicated. The large-scale structure of magnetic fields derived from pulsar RMs are indicated by thick arrows. See Han et al. (2006) for details. RMs of extragalactic radio sources of $|b|<8 \mathrm{deg}$ (data from Clegg et al. 1992, Gaensler et al. 2001, Brown et al. 2003, Brown et al. 2007) are also displayed in the outskirt ring. Positive RMs are shown by filled circles and negative RMs by open circles. The RM limits of $\pm 1000 \mathrm{rad} \mathrm{m}^{-2}$ are set at the outer and inner edges of the ring. As one can see from this plot, we have not many measurements for the magnetic fields for one half of the Galactic disk. The RMs of extragalactic radio sources become scarcer and scarcer in the region of $|l|<45 \mathrm{deg}$.

direct estimate of the strength of the field through normalization by the dispersion measure (DM). The RM is defined by $\phi=\mathrm{RM} \lambda^{2}$, where $\phi$ is the position angle in radians of linearly polarized radiation relative to its infinite-frequency $(\lambda=0)$ value and $\lambda$ is its wavelength (in $\mathrm{m}$ ). For a pulsar at distance $D$ (in pc), the RM (in radians $\mathrm{m}^{-2}$ ) is given by $\mathrm{RM}=0.810 \int_{0}^{D} n_{e} \mathbf{B} \cdot d \mathbf{l}$, where $n_{e}$ is the electron density in $\mathrm{cm}^{-3}, \mathbf{B}$ is the vector magnetic field in $\mu \mathrm{G}$ and $d \mathbf{l}$ is an elemental vector along the line of sight toward us 
(positive RMs correspond to fields directed toward us) in pc. With the DM $=\int_{0}^{D} n_{e} d l$, we obtain a direct estimate of the field strength weighted by the local free electron density

$$
\left\langle B_{\|}\right\rangle=\frac{\int_{0}^{D} n_{e} \mathbf{B} \cdot d \mathbf{l}}{\int_{0}^{D} n_{e} d l}=1.232 \frac{\mathrm{RM}}{\mathrm{DM}} .
$$

where RM and DM are in their usual units of $\operatorname{rad~m}^{-2}$ and $\mathrm{cm}^{-3} \mathrm{pc}$ and $B_{\|}$is in $\mu \mathrm{G}$.

Previous analysis of pulsar RM data has often used the model-fitting method (Han \& Qiao 1994, Indrani \& Deshpande 1999), i.e., to model magnetic field structures in all of the paths from the pulsars to us (observer) and fit them, together with the electron density model, to the observed RM data. Significant improvement can be obtained when both RM and DM data are available for many pulsars in a given region with similar lines of sight. Measuring the gradient of RM with distance or DM is the most powerful method of determining both the direction and magnitude of the large-scale field local in that particular region of the Galaxy (Lyne \& Smith 1989, Han et al. 2002, Weisberg et al. 2004, Han et al. 2006). Field strengths in the region can be directly derived (instead of modeled) from the slope of trends in plots of RM versus DM. Based on Equation 2.1, we got

$$
\left\langle B_{\|}\right\rangle_{d 1-d 0}=1.232 \frac{\Delta \mathrm{RM}}{\Delta \mathrm{DM}}
$$

where $\left\langle B_{||}\right\rangle_{d 1-d 0}$ is the mean line-of-sight field component in $\mu \mathrm{G}$ for the region between distances $d 0$ and $d 1, \Delta \mathrm{RM}=\mathrm{RM}_{d 1}-\mathrm{RM}_{d 0}$ and $\Delta \mathrm{DM}=\mathrm{DM}_{d 1}-\mathrm{DM}_{d 0}$.

So far, RMs of 550 pulsars have been observed (Hamilton \& Lyne 1987, Qiao et al. 1995, Han et al. 1999, Weisberg et al. 2004, Han et al. 2006). Most of the new measurements are for the fourth and first Galactic quadrants where sources are relatively distant. This enables us to investigate the structure of the Galactic magnetic field over a much larger region than was previously possible. We detected counterclockwise magnetic fields in the most inner arm, the Norma arm (Han et al. 2002). A more complete analysis for the fields near the tangential regions of most probable spiral of our Galaxy (Han et al. 2006) gives a similar picture for the coherent large-scale fields aligned with the spiral-arm structure in the Galactic disk, as shown in Fig.1: magnetic fields in all inner spiral arms are counterclockwise when viewed from the North Galactic pole. On the other hand, at least in the local region and in the inner Galaxy in the fourth quadrant, there is good evidence that the fields in interarm regions are similarly coherent, but clockwise in orientation. There are at least two or three reversals in the inner Galaxy, probably occurring near the boundary of the spiral arms. The magnetic field in the Perseus arm cannot be determined well. The negative RMs for distant pulsars and extragalactic sources (see Fig. 1) in fact suggest the interarm fields both between the Sagittarius and Perseus arms and beyond the Perseus arm are predominantly clockwise.

The fluctuations in the RM distribution of extragalactic radio sources (Clegg et al. 1992, Gaensler et al. 2001, Brown et al. 2003, Brown et al. 2007) with Galactic longitude, especially these of the fourth Galactic quadrant, are consistent with magnetic field directions derived from the tangential regions of the arms (see Fig. 1). This implies that the dominant contribution of RMs of extragalactic radio sources behind the Galactic disk comes from the interstellar medium mainly in tangential regions. However, modeling the averaged RM distribution of scattered RM data of background radio sources behind the Galactic disk requires fewer field reversals (Brown et al. 2007) maybe due to the smearing effect in the box-car averaging process over $9^{\circ}$ bins along the Galactic longitude, larger than the separation between the inner arms. 
With much more pulsar RM data now available, Han et al. (2006) were able to measure, rather than model, the regular field strength near the tangential regions in the 1st and 4th Galactic quadrants, and then plot the dependence of regular field strength on the Galacto-radii. Although the "uncertainties", which in fact reflect the random fields, are large, the tendency is clear that fields get stronger at smaller Galactocentric radii and weaker in interarm regions. To parameterize the radial variation, an exponential function was used. This was chosen to give the smallest $\chi^{2}$ value and to avoid a singularity at $R=0$ (for $1 / R$ ) and unphysical values at large $\mathrm{R}$ (for the linear gradient). The function is, $B_{\text {reg }}(R)=B_{0} \exp \left[\frac{-\left(R-R_{\odot}\right)}{R_{\mathrm{B}}}\right]$, with the strength of the large-scale or regular field at the Sun, $B_{0}=2.1 \pm 0.3 \mu \mathrm{G}$ and the scale radius $R_{\mathrm{B}}=8.5 \pm 4.7 \mathrm{kpc}$.

\section{Galactic magnetic fields on small scales}

Small-scale magnetic fields can be revealed by polarization surveys of diffuse radio emission of the Galactic plane, and by polarization observations of molecular clouds and supernova remnants by either linear polarization mapping or Zeeman splitting of line emission or maser emission. The statistics of these observations and the statistics of RMs of pulsars or extragalactic radio sources give the overall properties of the small-scale magnetic fields.

Polarization surveys of the Galactic plane have been comprehensively reviewed by Reich (2007). See references therein. Here I would like to mention that the observed polarized emission of the Galactic plane often has a scale size of about tens or hundreds of pc, and is the sum of all contributions coming from various regions along a line of sight with different polarization properties (i.e. polarization angle and polarization percentage) at different distance. Emission from more distant regions suffers from more Faraday effect produced by foreground interstellar medium. If the emission brightness at various distances are more or less similar, then the observed polarized emission is predominantly from local regions. Accumulating polarized emission from different regions should "depolarize" each other. This is more obvious in lower frequencies. Note that disordered polarized structures smaller than an observational beam could be smeared out by the so-called beam depolarization. Therefore, observations with a smaller beam would detect more polarized structures. Observations at higher frequencies should show structures at larger distances. These polarized structures are closely related to the magnetic field structure where the emission is generated. The Sino-German $6 \mathrm{~cm}$ polarization survey (Sun et al. 2007) of the Galactic plane using the Urumqi 25m telescope is currently been carried out, and we have detected some magnetic structures from Faraday screens and supernova remnants, some of which would not be detectable at lower frequencies (Sun et al. 2007, $\mathrm{Xu}$ et al. 2007).

In recent years, with development of instruments and backend technology, a lot of molecular clouds have been directly mapped for polarized emission at mm, submm or infrared bands, some by single dish, some by interferometers (see review by Heiles \& Crutcher 2005). These maps always show field orientation on a scale of the cloud size, i.e. a scale of pc to tens of pc. I do not have to cover this topic as readers can find comprehensive information from the reviews given by Crutcher (2007) and Vlemmings (2007) in this volume.

Some maser spots have a very small scale size, of AU rather than pc. From star formation regions or HII regions, the Zeeman splitting of the maser lines can directly gives the field strength in situ as well as the field direction in the line of sight. In recent years, there have been many new measurements, e.g. VLBA measurements by Fish et al. 


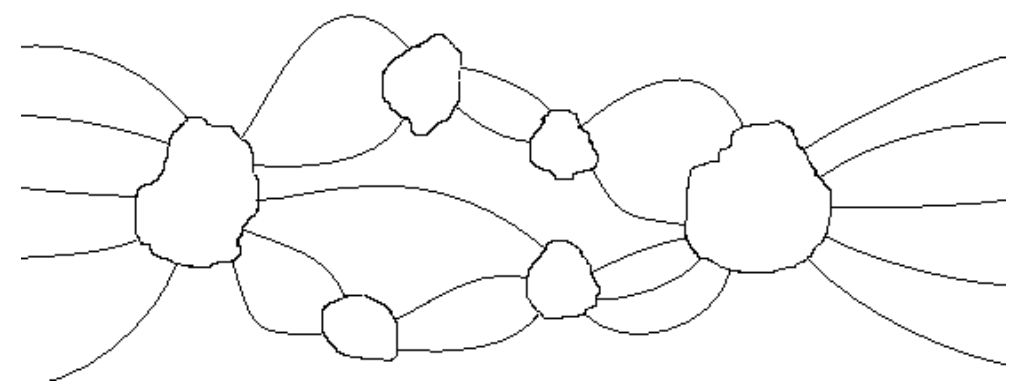

Figure 2. The magnetic fields on small scales, e.g. in clouds, are possibly related to the large-scale fields. See also Beck et al. (1991).

(2006) or other measurements by Hutawarakorn \& Cohen (2005). See Han \& Zhang (2007) for a list of all previous measurements. It is amazing that magnetic fields on such a small scale are possibly related to the large-scale Galactic magnetic fields (see below).

One important tool for studying small-scale magnetic fields is to use statistics. Detailed statistics for polarization survey data of the Galactic plane have been rare, but would be very useful. Statistics of the mean deviations of "polarization vectors" for clouds have been used to estimate the field strength in the cloud (see e.g. Crutcher et al. 2004). Minter \& Spangler (1996) have worked out the structure function of a group of RMs of extragalactic radio sources in a mid-latitude region, showing that the magnetic fields probably follow the Kolmogorov spectrum for a scale less than a few pc, while it becomes flatter above a few pc up to 80 pc, maybe due to the thin shape of the Galactic disk. Sun \& Han (2003) found that the structure function for RMs at the two Galactic poles are flat, indicating the random RM distribution, but at lower latitudes it becomes inclined with different slopes at different Galactic longitudes. Haverkorn et al. (2006) found that the structure functions of RMs in the arm tangential directions have much larger slopes than those in the interarm directions, indicating that the arm regions are more turbulent. The outer scale is as small as only 17 pc. Han et al. (2004) used statistics of pulsar RMs in a very large region of the two third of the Galactic disk, and obtained a flat magnetic energy spectrum for scales between $500 \mathrm{pc}$ and $15 \mathrm{kpc}$, which is different from the Kolmogorov spectrum at small-scales and should constrain the theoretical simulations (e.g. Balsara \& Kim 2005) on how the Galactic magnetic fields were generated and maintained.

\section{The connection of magnetic fields on large and small scales}

On one hand, molecular clouds were formed by contraction of diffuse gas in the interstellar medium, and the magnetic fields are so enhanced that they have the same energy as the kinetic energy (Crutcher 1999). Magnetic fields of clouds have been observed to have an hourglass shape, which is an indication of field direction conservation in the ISM during the contraction (see Fig. 2 and Beck et al. 1991). On the other hand, magnetic fields in the shell of supernova remnants are also enhanced in ISM due to supernova explosions, as can be seen from the polarized emission of remnants. Magnetic fields at this scale, i.e. the energy injection scale into ISM from the kinetic, should be the strongest. On average, random magnetic fields are stronger than the large-scale fields (Rand \& Kulkarni 1989, Han \& Qiao 1994, Heiles 1996b, Han et al. 2004).

However, evidence emerges for a possible relationship between the field directions of large-scale and small-scale magnetic fields. Recently, the observations ( $\mathrm{Li}$ et al. 2006) have shown that the majority JinLin do you mean 'majority' or something else? of magnetic 
field orientations of molecular clouds seem to be preferentially parallel to the Galactic plane. From the radio observations of supernova remnants, the bilateral remnants always tend to have the longer axis parallel to the Galactic plane (Gaensler 1998). This could be understandable given the fact that the Galactic magnetic fields are confined to the Galactic plane, and the strongest component of the Galactic magnetic fields is the azimuthal component, much stronger than the radial and vertical components (Han \& Qiao 1994, Han et al. 1999).

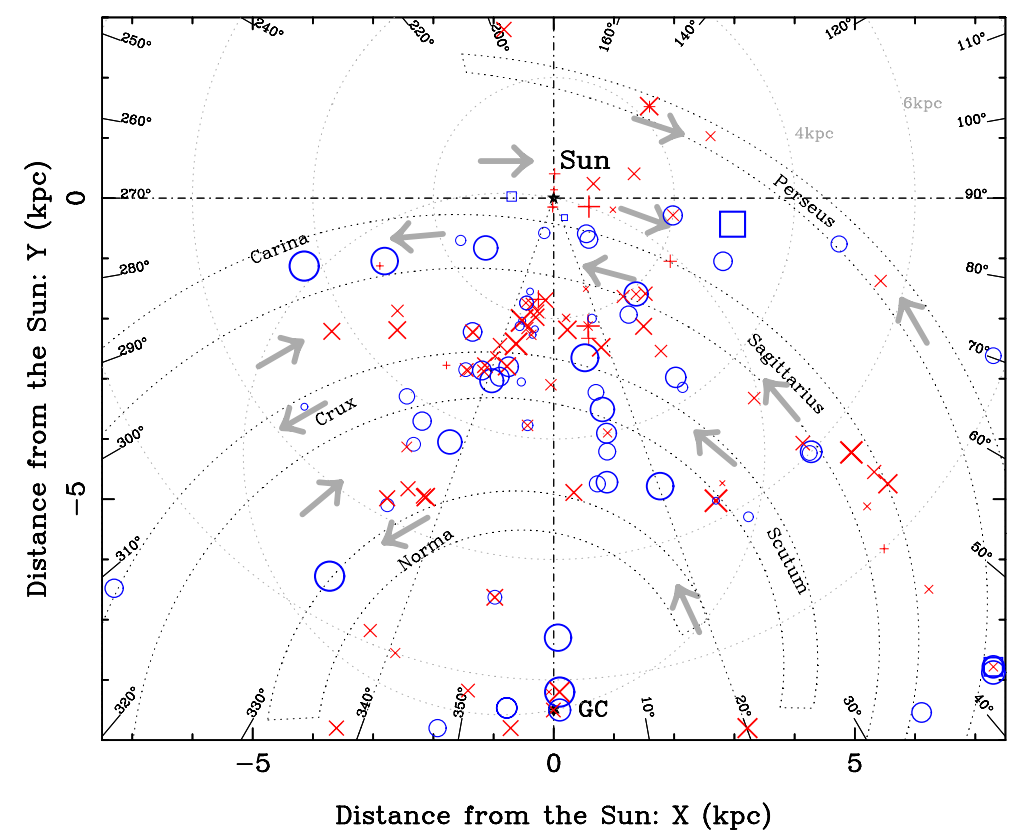

Figure 3. The medians of field measurements from Zeeman splitting of $\mathrm{OH}$ masers (cross and circles) in 137 objects or $\mathrm{HI}$ or $\mathrm{OH}$ lines of 17 molecular clouds (plus and squares) projected onto the Galactic plane, with the rough indication of spiral arms and the magnetic field directions (arrows) derived from pulsar RM data. The linear sizes of the symbols are proportional to the square root of the field-strength values, with limits of 0.5 and $9 \mathrm{mG}$ for fields from the median maser fields and of $15 \mu \mathrm{G}$ and $270 \mu \mathrm{G}$ for median cloud fields. The crosses or pluses on the right $(0 \mathrm{deg}<l<180 \mathrm{deg})$ represent positive $B$, i.e. the field direction going away from observer, and circles or squares going towards us. The symbols on the left (180 deg $<l<360 \mathrm{deg})$ are reversed, so that all crosses and pluses are consistent with the clockwise fields viewed from the Northern Galactic pole, and all circles and squares with counterclockwise fields. See Han \& Zhang (2007) for details.

If the magnetic fields in molecular clouds are preserved from the permeated magnetic fields in the interstellar medium during cloud formation, then the question arises as to whether or not the magnetic fields in molecular clouds can still "remember" the largescale magnetic fields in the interstellar medium? Are they sufficiently strong that their correlation with the large-scale fields was not destroyed by turbulence in clouds? If the answer is yes, then observations of cloud fields could be an independent approach to reveal the large-scale structure of the Galactic magnetic fields.

Han \& Zhang (2007) collected measurements of the magnetic fields in molecular clouds, including Zeeman splitting data of $\mathrm{OH}$ masers in clouds of $\mathrm{HII}$ regions and $\mathrm{OH}$ or $\mathrm{HI}$ absorption or emission lines of clouds themselves. The data show clear structures in the sign distribution of the median of line-of-sight components of the magnetic field (see Fig. 3). Compared to the large-scale Galactic magnetic fields derived from pulsar RMs, 
the sign distribution of the Zeeman data shows similar large-scale field reversals. We conclude that the magnetic fields in the clouds may still "remember" the directions of magnetic fields in the Galactic ISM to some extent, and could be used as complementary tracers of the large-scale Galactic magnetic structure.

How can such coherent magnetic field directions occur from low density $\left(\sim 1 \mathrm{~cm}^{-3}\right)$ to higher density $\left(\sim 10^{3} \mathrm{~cm}^{-3}\right)$ ISM clouds, even to the highest density maser regions $\left(\sim 10^{7} \mathrm{~cm}^{-3}\right)$, after a density compression of about 3 , or even 10 , orders of magnitude? One implication of this result is that the clouds probably do not rotate much after they are formed as otherwise the field directions of clouds we measured would be random. During the process of star formation, the clouds seem to be too heavy to be rotated, although there are jets or disks from newly formed stars which may have some dynamic effects. Furthermore, the fields in the molecular clouds are strong enough after the contraction that the turbulence in the clouds cannot significantly alter the magnetic field status.

\section{Conclusions}

Magnetic fields have been observed on all scales in our Galaxy. However, our knowledge of Galactic magnetic fields and their impact on ISM physics is far from complete. Large-scale magnetic fields in some regions have been delineated by enriched pulsar RM data(Han et al. 2006). However, large-scale magnetic fields in many regions remain to be measured with more pulsar RM data or probed by extragalactic radio sources (Brown et al. 2007). Small-scale fields have been measured in several ways: from polarization surveys of the Galactic plane (tens pc or hundreds pc), polarization mapping of clouds and supernova remnants ( $\mathrm{pc}$ or tens $\mathrm{pc}$ ), using the structure function of RMs (pc or tens $\mathrm{pc}$ ), and Zeeman splitting observations of line emissions (AU to pc). Although smallscale magnetic fields appear as random or "noise" on the larger-scale, and are stronger than the large-scale magnetic fields, observational evidence already shows some physical connections between the small scale magnetic fields and the large-scale magnetic fields.

\section{Acknowledgements}

I am very grateful to colleagues who have collaborated with me: Dr. R.N. Manchester from Australia Telescope National Facility, CSIRO, Prof. G.J. Qiao from Peking University (China), Prof. Andrew Lyne from Jodrell Bank Observatory (UK), and Dr. Katia Ferriére from Observatory of Midi-Pyrénées (France), Dr. JiangShui Zhang from GuangZhou University (China), Dr. Willem van Straten from Swinborne University (AU). The author is supported by the National Natural Science Foundation of China (10521001 and 10473015).

\section{References}

Andreasyan, R. R. \& Makarov, A. N. 1989, Afz, 31, 247

Balsara, D. \& Kim, J. 2005, ApJ 634, 390

Beck R. 2005, In: Magnetic Fields in the Universe: From Laboratory and Stars to Primordial Structures, AIP 784, p.343

Beck, R., Berkhuijsen, E. M., \& Bajaja, E., 1991, IAU Sypm. 146, p.209

Beck R., Brandenburg A., Moss D., Shukurov A. M., \& Sokoloff D. D., 1996, ARA\&A, 34, 155

Boulares, A. \& Cox, D. 1990, ApJ, 365, 544

Brown, J. C., Taylor, A. R., \& Jackel, B. J. 2003, ApJS, 145, 213

Brown, J. C., Haverkorn, M., Gaensler, B. M., \& et al. 2007, ApJ, in press

Chuss, D. T., Davidson, J. A., Dotson, J. L., \& et al. 2003, ApJ, 599, 1116

Clegg, A. W., Cordes, J. M., Simonetti, J. M., Kulkarni, S. R. 1992, ApJ, 386, 143 
Crutcher, R. M. 1999, ApJ, 520, 706

Crutcher, R. M. 2007, IAU Symp.242, this volumn.

Crutcher, R. M., Nutter, D. J., Ward-Thompson, D., \& Kirk, J. M. 2004, ApJ, 600, 279

Duncan, A. R., Haynes, R. F., Jones, K. L., \& Stewart, R. T. 1997, MNRAS, 291, 279

Duncan, A. R., Haynes, R. F., Reich, W., Reich, P., \& Gray, A. D. 1998, MNRAS, 299, 942

Duncan, A. R., Reich, P., Reich, W., \& Fürst, E. 1999, A\&A, 350, 447

Fish, V. L., Reid, M. J., Agron, A. L. \& Menten, K. M., 2003, ApJ, 596, 328

Gaensler, B. M. 1998, ApJ, 493, 781

Gaensler, B. M., Dickey, J. M., McClure-Griffiths, N. M., \& et al. 2001, ApJ, 549, 959

Hamilton, P. A. \& Lyne, A. G. 1987, MNRAS, 224, 1073

Han, J. L. \& Qiao, G. J. 1994, A\&A, 288, 759

Han, J. L., Ferriere, K., \& Manchester, R. N. 2004, ApJ, 610, 820

Han, J. L., Manchester, R. N., Berkhuijsen, E. M., \& Beck, R. 1997, A\&A, 322, 98

Han, J. L., Manchester, R. N., \& Qiao, G. J. 1999, MNRAS, 306, 371

Han, J. L., Manchester, R. N., Lyne, A. G., \& Qiao, G. J. 2002, ApJ, 570, L17

Han, J. L., Manchester, R. N., Lyne, A. G., Qiao, G. J., \& van Straten, W. 2006, ApJ, 642, 868.

Han, J. L., \& Zhang, J. S. 2007, A\&A, 464, 609

Haverkorn, M., Gaensler, B. M., Brown, J. C., et al. 2006, ApJ 637, L33

Heiles, C. 1996a, ApJ, 462, 316

Heiles, C. 1996b, in ASP Conf. Ser. 97: Polarimetry of the Interstellar Medium, 457

Heiles, C, \& Crutcher, R. 2005, In: Cosmic Magnetic Fields, LNP 664, 137

Haynes, R. F., Stewart, R. T., Gray, A. D., \& et al. 1992, A\&A, 264, 500

Hutawarakorn, B. \& Cohen, R. J., 2005, MNRAS, 357, 338

Indrani, C. \& Deshpande, A. A. 1999, New Astronomy, 4, 33

Junkes, N., Fürst, E., \& Reich, W. 1987, A\&AS, 69, 451

LaRosa, T. N., Nord, M. E., Lazio, T. J. W., \& Kassim N. E. 2004, ApJ, 607, 302

LaRosa, T. N., Brogan C. L., Shore S. N., et al. 2005, ApJ, 626, L23

Li, H., Griffin, G. S., Krejny, M., \& et al. 2006, ApJ, 648, 340

Lyne, A. G. \& Smith, F. G. 1989, MNRAS, 237, 533

Mathewson, D. S. \& Ford, V. L. 1970a, MemRAS, 74, 139.

Minter, A. H. \& Spangler, S. R. 1996, ApJ, 458, 194

Morris, M., Uchida, K.,\& Do, T. 2006, Nature, 440, 308

Nord, M. E., Lazio, T. J. W., Kassim, N. E., et al. 2004, AJ, 128, 1646

Novak, G., Chuss, D. T., Renbarger, T., \& et al. 2003, ApJ, 583, L83

Prouza, M. \& Smída, R. 2003, A\&A 410, 1

Qiao, G. J., Manchester, R. N., Lyne, A. G., \& Gould, D. M. 1995, MNRAS, 274, 572

Rand, R. J. \& Kulkarni, S. R. 1989, ApJ, 343, 760

Reich, W. 2007, in: Cosmic Polarization, (astro-ph/0603465), p. 91

Reich, W., Fürst, E., Reich, P., et al. 2002, in AIP Conf. Proc. 609: p. 3

Roy, S., Rao, A. P., Subrahmanyan, R. 2005, MNRAS 360, 1305

Simard-Normandin, M. \& Kronberg, P. P. 1980, ApJ, 242, 74

Sun, X. H. \& Han, J. L. 2004, in: Magnetized Interstellar Medium, p. 25

Sun, X. H., Han, J. L., Reich, W., \& et al. 2007, A\&A 463, 993

Tinyakov, P. G. \& Tkachev, I. I. 2002, Astropart. Ph. 18, 165

Vlemming, W. 2007, IAU Symp. 242, this volume

Weisberg, J. M., Cordes, J. M., Kuan, et al. 2004, ApJS, 150, 317

Xu, J. W., Han, J. L., Reich, W., \& et al. 2007, A\&A, in press

Yusef-Zadeh, F., \& Morris, M. 1987, ApJ, 320, 545

Yusef-Zadeh, F., Hewitt, J. M., \& Cotton, W. 2004, ApJS, 155, 421 